\title{
Temsirolimus for patients with metastatic renal cell carcinoma: outcomes in patients receiving temsirolimus within a compassionate use program in a tertiary referral center
}

\author{
This article was published in the following Dove Press journal: \\ Drug Design, Development and Therapy \\ 17 December 2014 \\ Number of times this article has been viewed
}

\author{
Mehran Afshar ${ }^{1, *}$ \\ Jennifer Pascoe ${ }^{1,2, *}$ \\ Sue Whitmarsh' \\ Nicholas James ${ }^{1,3}$ \\ Emilio Porfiri ${ }^{1,2}$ \\ 'Queen Elizabeth Hospital \\ Birmingham NHS Foundation Trust, \\ ${ }^{2}$ Cancer Research UK Clinical Trials \\ Unit, School of Cancer Sciences, \\ University of Birmingham, Birmingham, \\ UK; ${ }^{3}$ Cancer Research Unit, Clinical \\ Trials Unit, University of Warwick, \\ Coventry, UK \\ *These authors have contributed \\ equally to this work
}

\begin{abstract}
Aim: Temsirolimus has shown efficacy as first-line treatment of patients with metastatic renal cell carcinoma and poor prognostic features. The efficacy of temsirolimus in other clinical settings, such as second-line therapy, is unclear. The aim of this study was to investigate the outcomes of an unselected group of patients with renal cancer treated with temsirolimus in a compassionate use program.
\end{abstract}

Patients and methods: This retrospective analysis included all patients receiving temsirolimus at a tertiary referral center between November 2007 and October 2008. Information was obtained through review of patient notes, electronic records, and pharmacy records. Baseline characteristics, prognostic features, and previous treatments were recorded for all patients. Outcome measures were response rate, progression-free survival (PFS), overall survival (OS), and toxicities.

Results: Thirty-eight patients were included in the analysis, with median age of 62 years, among whom $37 \%$ were untreated and $63 \%$ had received one or more previous treatments. Thirty-four percent of the patients had three or more poor prognostic factors. Four patients (11\%) achieved a partial response (PR); in all four of these patients, the PR was confirmed by two subsequent computed tomography (CT) scans, and in one patient, the PR lasted for more than 18 months. A total of $34 \%$ achieved stable disease, and $50 \%$ had disease progression. Median OS was 7.6 months (95\% confidence interval [CI] 4.8-10.5), and median PFS was 3.2 months (95\% CI 1.0-5.5). Patients with two or fewer poor prognostic factors had a survival of 10.12 months compared with 5.03 months of those with three or more. Median survival was 14.9 months for untreated patients and 6.4 months for previously treated patients.

Conclusion: Our results indicate some efficacy of temsirolimus in untreated patients with renal tumors and poor-intermediate prognosis, although the limitations of small sample size and retrospective nature must be taken into account. The role of temsirolimus in previously treated patients remains controversial given the recently published results of the INTORSECT trial and the discrepancies between the few published series.

Keywords: survival, renal cancer, toxicity, retrospective, tumor

\section{Introduction}

The management of metastatic renal cell carcinoma (RCC) has been revolutionized by the advent of a new generation of drugs that target the molecular pathways often implicated in the development of this malignancy. ${ }^{1}$ Drugs targeting the aberrant activation of the vascular endothelial growth factor (VEGF) pathway, such as sunitinib, pazopanib, and bevacizumab, have shown significant efficacy and have replaced interferon-based
Correspondence: Emilio Porfiri

Cancer Research UK Clinical Trials Unit, School of Cancer Sciences, University of Birmingham, Vincent Drive, Edgbaston, Birmingham BI5 2TT, UK

Tel +447768 838762

Email emilio.porfiri@uhb.nhs.uk 
immunotherapy in the first-line treatment of metastatic RCC. ${ }^{2}$ Another promising group of agents are the inhibitors of mammalian target of rapamycin (mTOR) kinase: temsirolimus and everolimus. ${ }^{3}$ The mTOR kinase is a component of intracellular signaling pathways regulating cell proliferation, nutrient uptake, and the response to hypoxic stress. A Phase II study suggested that temsirolimus is active in advanced RCC, especially in patients with poor prognosis. ${ }^{4}$ A pivotal Phase III trial compared temsirolimus with interferon- $\alpha$ and with a combination of the two in untreated RCC patients who had at least three of six risk factors for short survival. ${ }^{5}$ Poor performance status (PS) was one of the six risk factors and was present in more than $80 \%$ of the patients in this study. The trial showed that the patients randomized to receive temsirolimus had a median survival of 10.9 months $(95 \%$ confidence interval [CI] 6.1-8.8) compared with 7.3 months (95\% CI 6.1-8.8) for the patients receiving interferon- $\alpha$ alone (hazard ratio $[\mathrm{HR}]=0.73,95 \% \mathrm{CI} 0.58-0.92, P=0.008$ ). The patients treated with the combination of interferon- $\alpha$ and temsirolimus did not have a survival advantage compared with the patients receiving interferon- $\alpha$ alone. Based on the results of this trial, temsirolimus has become the standard of care for patients with advanced RCC and poor prognostic features. Importantly temsirolimus remains the only therapeutic option for this group of patients who were excluded from most trials of VEGF-targeted therapies and may not derive a benefit from treatment with these drugs. Currently a UK Clinical Research Network study is underway looking at pazopanib efficacy and safety in patients with advanced clear-cell RCC and Eastern Cooperative Oncology Group (ECOG) PS 2. This study may help determine the efficacy of pazopanib in this setting, but until those results are, available tyrosine kinase inhibitors are not indicated for patients with an ECOG PS $\geq 2 .^{6}$

Because of the different mechanism of action, temsirolimus was also used outside of the licensed indication in RCC patients who failed one or more lines of VEGF-targeted therapy. ${ }^{7,8}$ Only data from retrospective series had been published at the time of this study; nevertheless the data suggested that second-line treatment with temsirolimus may provide further clinical benefit to these patients.

Between November 2007 and October 2008 temsirolimus was available in the UK within a compassionate use program wherein the drug was provided for free, on compassionate grounds, by the drug manufacturer. Thirty-eight patients received temsirolimus within this program at our center. In this paper we present the treatment details and outcomes of this cohort.

\section{Patients and methods}

We retrospectively reviewed clinical annotations, pharmacy records, and radiology files of 38 consecutive patients with advanced RCC who commenced treatment with temsirolimus in our center between November 2007 and October 2008. The final date of data collection was January 31, 2010. The drug was provided by the manufacturer within a compassionate use program, ahead of European Medicines Agency approval. Patients were selected from those undergoing treatment for RCC in our institution and those referred from other centers for consideration for entry into the program. Therapy with temsirolimus was offered to patients who, in the opinion of the attending physician, had the potential to benefit from this drug, in particular, patients who met the eligibility criteria of the Phase III clinical trial. In addition, a number of patients who had progressed after VEGF-targeted therapy and patients for whom treatment was not available from the UK National Health Service were also included in the program. All patients had adequate organ function and provided written informed consent.

Patients were stratified according to the number of adverse prognostic factors, as described in the pivotal Phase III trial. ${ }^{5}$ Unfortunately we were unable to include lactate dehydrogenase (LDH) in the prognostic factor analysis as LDH was not routinely tested in our center. Therefore only the following five adverse prognostic factors were taken into account: ECOG PS $\geq 2$, hemoglobin level below lower limit of normal $(<13.5 \mathrm{~g} / \mathrm{dL}$ for males, or $<11.5 \mathrm{~g} / \mathrm{dL}$ for females); corrected $\mathrm{Ca}^{2+}$ level $>2.6 \mathrm{mmol} / \mathrm{L}$; three or more metastatic sites; and time from diagnosis to treatment with temsirolimus $<1$ year. ${ }^{9}$ Patients were also stratified according to the presence of previous systemic treatments.

Temsirolimus was administered weekly in an outpatient setting at a dose of $25 \mathrm{mg}$ intravenously. Adverse events and laboratory abnormalities were classified according to Common Terminology Criteria for Adverse Events version 4.0. Treatment-related toxicity determined to require an intervention was addressed with supportive therapy and when necessary, with a dose reduction, a treatment delay, or both. Data regarding the incidence of toxicity were collected from clinical annotations and electronic patient records. Patients were reviewed weekly during the 1 st month of treatment, then on a four-weekly basis. Routine monitoring of patients comprised weekly assessment of blood counts, creatinine, urea and electrolytes, liver function tests. Fasting blood glucose and lipid levels were not routinely analyzed. 
Data collected included baseline clinical characteristics, details of previous treatments, outcome of temsirolimus therapy, and toxicity. Treatment outcome was assessed by analyzing objective response rate, overall survival (OS), and progression-free survival (PFS).

Response to treatment was assessed radiologically in all the patients who had received at least eight weekly doses of temsirolimus; computed tomography (CT) scans were then repeated every 3 months or more frequently if clinically indicated. The Response Evaluation Criteria in Solid Tumors (RECIST 1.0) ${ }^{10}$ were used to assess tumor response. Scans were routinely reported by the Radiology Department and reviewed by the radiologists of the hospital Urology-Oncology multidisciplinary team. Response rate, as the percentage of patients with a partial or complete response, was calculated. OS was measured from the date of commencement of temsirolimus until the date of death by any cause; patients were censored at the date last known to be alive. PFS was calculated from the start of the treatment to the earliest evidence of disease progression (radiological or clinical) or death by any cause; patients were censored at the last time known to be progression-free. The KaplanMeier product-limit method was used to estimate OS and PFS. Given the small sample size, these estimates should be considered illustrative rather than definitive.

\section{Results \\ Patients}

Thirty-eight patients who commenced treatment with temsirolimus between November 2007 and November 2008 were included in the study. Median age was 62 years and $68 \%$ were males. Two-thirds of the patients had clear cell RCC, four patients had a papillary RCC, seven were diagnosed with unclassified RCC, and two had a clinical diagnosis of RCC. Thirteen patients (35\%) had a poor PS (ECOG 2-3), and 23 patients $(61 \%)$ more than two metastatic sites. Thirteen patients $(34 \%)$ had three or more poor prognostic factors (Table 1).

Fourteen patients $(37 \%)$ were untreated, whereas 24 patients $(63 \%)$ had received previous systemic treatments: 14 patients had one previous treatment (nine with interferon- $\alpha$, three with sunitinib, and two with sorafenib); ten patients had two previous treatments (seven with interferon followed by sunitinib, two with interferon followed by cediranib, and one with interferon followed by sorafenib). One patient had received interferon followed by sorafenib then sunitinib. Almost $90 \%$ of the patients had undergone a nephrectomy, either radical or cytoreductive.
Table I Poor prognostic factors at baseline

Patients, n (\%)

\begin{tabular}{ll} 
Poor prognostic factors & \\
ECOG PS $\geq 2$ & $13(34 \%)$ \\
Time from diagnosis $<$ I year & $9(24 \%)$ \\
Greater than 2 metastatic sites & $23(61 \%)$ \\
Hemoglobin below normal limit & $23(61 \%)$ \\
High corrected serum calcium* & $9(24 \%)$ \\
Number of poor prognostic factors & \\
0 & $2(6 \%)$ \\
I & $10(26 \%)$ \\
2 & $13(34 \%)$ \\
3 & $7(18 \%)$ \\
4 & $5(13 \%)$ \\
5 & $1(3 \%)$ \\
\hline
\end{tabular}

Note: *Data not available in one patient.

Abbreviations: ECOG, Eastern Cooperative Oncology Group; PS, performance status.

\section{Treatment delivery}

A total of 1,112 doses of temsirolimus were administered to patients by the time of data censoring. The median number of treatments received per patient was 14 (range 3-129). Five patients were still on treatment at the time of data collection. Twelve patients received eight doses of treatment or less. Thirty-two patients (84\%) required at least one break from treatment. This was usually for toxicity but occasionally was due to personal circumstances. Thirteen patients (34\%) required at least one dose reduction (12 for toxicity, and one patient commenced treatment at a reduced dose due to age and frailty). Three patients ( $8 \%$ ) required a further dose reduction for ongoing toxicity.

\section{Toxicity}

Toxicity data was obtained through retrospective review of patients' notes, therefore data may not be exhaustive; nevertheless, it is expected that all drug-related and clinically significant adverse events were recorded by the attending physician. Data on laboratory abnormalities was collected from electronic laboratory records. Toxicity data is displayed in Table 2. The most common adverse events reported were rash (39\%) and mucositis (34\%). Drug-induced interstitial pneumonitis was observed in five (13\%) patients who were treated with corticosteroids and required a treatment break. All five patients fully recovered and were able to restart treatment. Many patients complained of asthenia and fatigue, which are known side effects of temsirolimus; however, given the poor prognosis and the advanced disease, it was difficult to determine whether these symptoms were diseaseor treatment-related. 
Table 2 Adverse events and laboratory abnormalities observed in the 38 patients

\begin{tabular}{lll}
\hline & $\begin{array}{l}\text { All grades, } \\
\mathbf{n}(\%)\end{array}$ & $\begin{array}{l}\text { Grade 3 and 4, } \\
\mathbf{n}(\%)\end{array}$ \\
\hline Adverse events & & \\
Skin rash - erythema & I5 (39) & I (3) \\
Mucositis & I3 (34) & $3(8)$ \\
Tiredness - fatigue & $8(2 \mathrm{I})$ & 0 \\
Pneumonitis & $5(13)$ & 0 \\
Peripheral edema & $4(10)$ & 0 \\
Diarrhea & $2(6)$ & 0 \\
Allergy & $2(6)$ & 0 \\
DVT & I (3) & I (3) \\
Nausea & I (3) & 0 \\
Myalgia & I (3) & 0 \\
Abdominal pain & I (3) & 0 \\
Laboratory abnormalities & & \\
Anemia & $37(97)$ & I (3) \\
Thrombocytopenia & 2 I (55) & 0 \\
Leukopenia & $6(16)$ & I (3) \\
Neutropenia & $3(8)$ & I (3) \\
Increased creatinine & $27(7 \mathrm{I})$ & $3(8)$ \\
Hypertriglyceridemia & $3(8)$ & I (3) \\
\hline Abrevation: DVT, deep &
\end{tabular}

Abbreviation: DVT, deep vein thrombosis.

The most frequently occurring laboratory abnormalities were anemia (97\%) and an elevated creatinine (71\%). Eight percent of patients had grade 3 or 4 elevation in serum creatinine, with one patient requiring temporary renal replacement therapy. In addition, although we did not routinely measure blood glucose, six (16\%) patients were noted to have a new diagnosis of diabetes or a worsening of diabetic control, and three (8\%) patients had elevated serum triglycerides (grade 4 in one patient). It is likely that routine measurement of blood glucose and lipids would have resulted in a higher incidence of hyperglycemia and hyperlipidemia.

\section{Efficacy}

\section{Response rate}

Response could not be evaluated in two patients (5\%) who stopped treatment early because of severe toxicity and did not undergo the scheduled response assessment. Four patients (11\%) achieved a partial response, 13 patients (34\%) stable disease, and 19 patients (50\%) had disease progression. In eight patients, disease progression was assessed clinically as a general deterioration or death occurred before the planned response assessment. Although the number of patients in our study is too small for subgroup analysis, our data seem to suggest that patients with three or more poor prognostic factors and patients who had received previous systemic treatments were more likely to develop disease progression than were patients with two or fewer poor prognostic factors or untreated patients.

\section{OS and PFS}

Median OS for the whole group of patients was 7.6 months (95\% CI 4.8-10.5); median PFS was 3.2 months (95\% CI 1.0-5.5). When we assessed survival according to number of poor prognostic risk factors, we found that patients with two or fewer risk factors had an OS of 10.1 months (95\% CI 3.7-16.5), and patients with three or more poor prognostic risk factors had an OS of 5.0 months $(95 \%$ CI 2.1-8.0) (Figure 1). PFS was 4.7 months (95\% CI 1.1-8.3) in the patients with two or fewer poor prognostic factors and was 2.6 months (95\% CI 2.0-3.1) for the patients with three or more poor prognostic factors. We also analyzed the survival according to whether patients had received previous systemic treatment. The median OS of the patients who had not received previous treatment was 14.9 months (95\% CI 2.4-27.4), and the median OS of the patients who had received one or more previous systemic treatments was 6.4 months (95\% CI 4.7-10.5) (Figure 2). Median PFS was 8.7 months (95\% CI 0-24.00) for untreated patients and 2.6 months (95\% CI 2.0-3.1) for previously treated patients. Among the 14 patients who had not received previous treatment, five (36\%) had three or more poor prognostic factors compared with eight (33\%) of the 24 patients who had received previous treatment.

\section{Discussion}

We retrospectively reviewed the clinical outcomes of a series of 38 patients with metastatic RCC who were treated with temsirolimus within a compassionate use program. The objective of the program was simply to provide treatment with temsirolimus ahead of marketing authorization to patients who might derive a benefit from it. Such loose entry criterion led to the recruitment of a patient population with diverse baseline characteristics. For example, only $34 \%$ of our patients had three or more poor prognostic features, and $63 \%$ had received at least one previous systemic treatment. Other centers participating in the temsirolimus compassionate use program employed a similar entry policy, thus including patients who had received previous systemic treatments as well as patients with good or intermediate prognosis..$^{12,13,15}$ Nevertheless, the mixed characteristics of the patients in these series were typical of patients seen in routine clinical practice and reflected the challenges of the day-to-day management of advanced RCC.

The median OS of our patients was 7.6 months, shorter than the survival of the patients treated with temsirolimus in the Phase III trial but also shorter than the survival observed in the other series of patients treated within the 


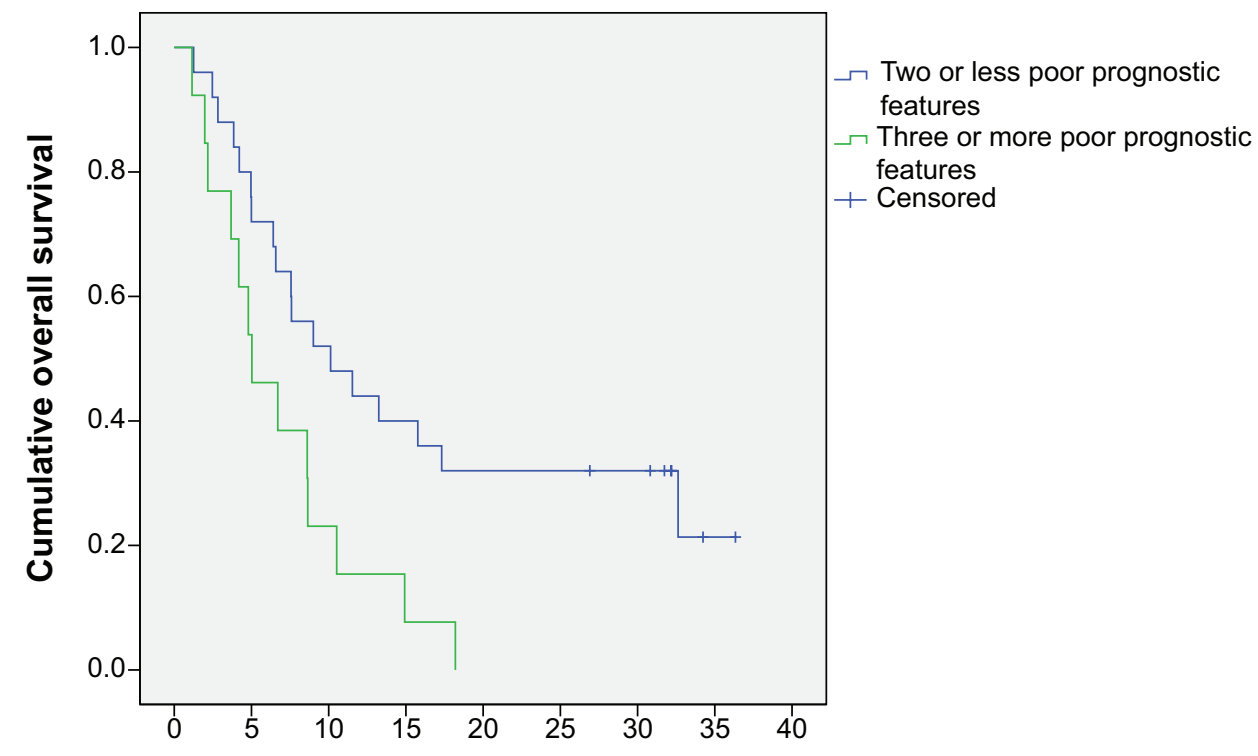

Time from commencing temsirolimus (months)

Figure I Kaplan-Meier plot of overall survival according to number of prognostic features.

compassionate use program. The diverse clinical characteristics, the small number of patients, and the retrospective nature of the study can perhaps account for such survival differences; nevertheless, $61 \%$ of our patients had more than two metastatic sites and 35\% had a poor PS, which may have influenced the survival outcome. When we analyzed the survival outcome according to the number of poor prognostic factors, we found that the patients who showed two or fewer poor prognostic factors had a median OS of 10.1 months (95\% CI 3.7-16.5) compared with 5 months (95\% CI 2.1-8.0) in the patients showing three or more poor prognostic factors. Unfortunately we were unable to include the LDH level in the risk factors analysis, therefore it is possible that a number of patients with three or more poor prognostic factors were included in the group of patients with up to two poor risk factors, thus affecting the survival data.

Twenty-four patients (63\%) who were treated with temsirolimus in the second- or third-line setting had a median OS of 6.1 months. By contrast, the median OS of the patients who had first-line temsirolimus was 14.9 months.

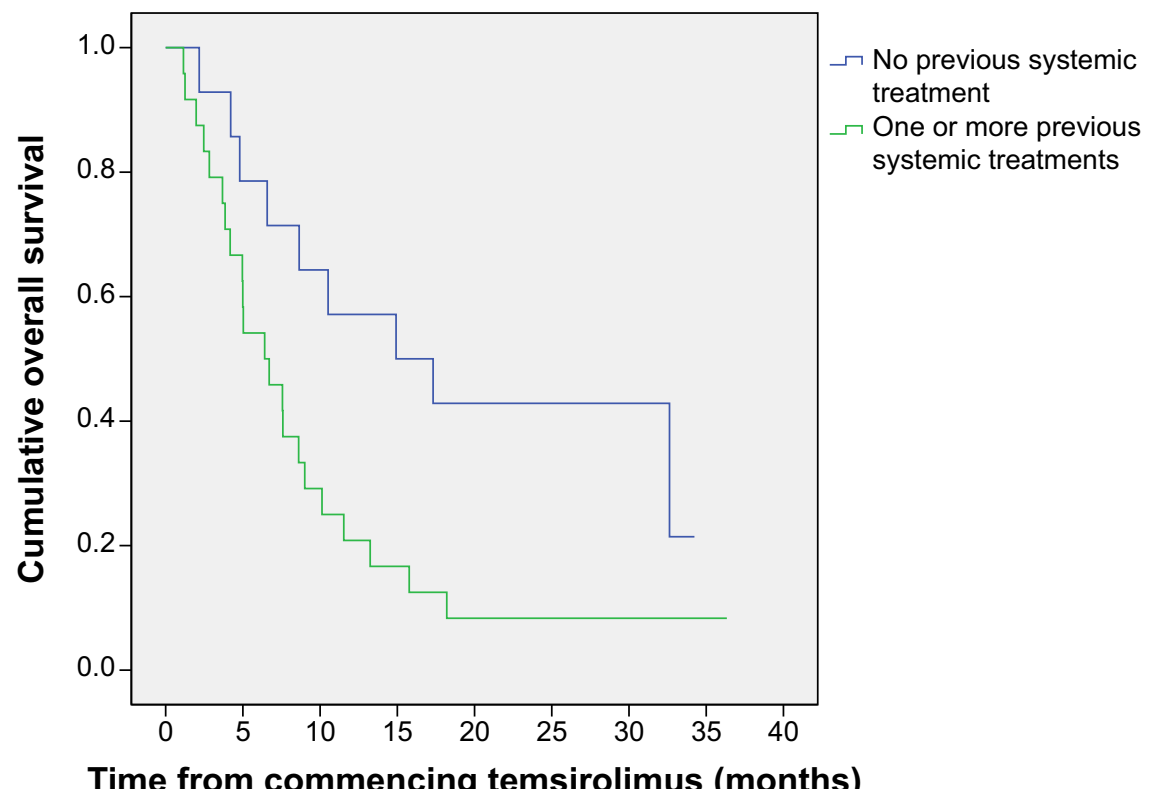

Figure 2 Kaplan-Meier plot of overall survival by previous treatment status. 
The number of patients was too small to formally subdivide each group according to prognostic status; however, $36 \%$ of the patients who had first-line temsirolimus and $50 \%$ of the patients who had previous systemic treatments showed three or more poor prognostic factors. Other authors who have used temsirolimus as a second-line therapy within the compassionate use program have reported better survival outcomes. MacKenzie et al observed a median OS of 11.2 months in 87 RCC patients who failed VEGF-targeted therapy. ${ }^{7}$ Lamm treated 30 RCC patients who had received on average two previous lines of therapy, and observed a median OS of 14.2 months. Weikert et al reported a median OS of 18 months in 29 patients treated after the failure of sunitinib and/or sorafenib. ${ }^{8}$ It is difficult to explain the discrepancy between our observations and the data from these series. The prognostic factor distribution in our patients was not dissimilar from that of the series published by MacKenzie et $\mathrm{al}^{7}$ but the small number of patients in our group could have affected the OS outcome. The majority of the patients in the series from Lamm were in the favorable or intermediaterisk group, which may explain the better survival data. In the series published by Weikert et al the prognostic factor distribution was not available, thus making it impossible to compare their results with other published work. ${ }^{8}$ Assessing the role of temsirolimus in the second-line setting is difficult due to the lack of clinical trial data and the discrepancies occurring between the small retrospective series that have been published. The results of a Phase III clinical trial comparing temsirolimus to sorafenib in patients who failed sunitinib were presented at the European Society of Medical Oncology Annual Meeting 2012. The INTORSECT Trial ${ }^{21}$ enrolled 512 patients with a PS of 0 or 1 and either clear cell or nonclear cell histology. The primary end point of PFS was prolonged by 4.28 months with temsirolimus compared with 3.91 months for sorafenib; however, this advantage was not statistically significant $(P=0.1933)$. More interestingly, a statistically significant advantage for sorafenib was observed in the secondary end point of OS. The median OS with temsirolimus was 12.27 months compared with 16.64 months with sorafenib ( $P=0.0144)$. However, an $8 \%$ RECIST 1.0 defined response rate was noted in both arms of the study.

The response rate we observed in our series was similar to that reported by other authors; nevertheless half of our patients had progressive disease as best response compared with a progressive disease rate of approximately $25 \%$ described in two other series. It is possible that such difference in response rate contributed to the worse survival achieved by our patients.
The treatment was sufficiently well tolerated. The toxicity observed was generally mild and expected. ${ }^{5,11}$ A significant rate of interstitial pneumonitis has recently been reported in the literature, ${ }^{13}$ and our observations serve to reinforce that clinicians should be alert to the possibility of a drug-induced pneumonitis when treating patients with temsirolimus. The most striking difference in the toxicity profile is the very high rate of elevation in serum creatinine, which we found in $71 \%$ of our patients, whereas elevation in serum creatinine was reported in only $14 \%$ of patients receiving temsirolimus in the Phase III trial ${ }^{5}$ and in $40 \%$ of the patients in the series published by Gerullis et al. ${ }^{11}$ The cause of the large number of patients experiencing increased creatinine is unclear. It should be noted though, that the majority of patients experienced only a grade 1 elevation that did not require any change in management.

Comparing clinical trial results with observations made in nonselected cohorts of patients from routine clinical practice is difficult but may provide information on the efficacy and toxicity of cancer drugs outside the trial setting. While in our series, the risk factor assessment was incomplete due to the lack of LHD measurements, our observations seem to confirm the role of temsirolimus in untreated RCC patients with poor-intermediate prognosis. Given the discrepancies in survival between retrospective series, the results of the of the Phase III trial of temsirolimus versus sorafenib have been awaited for clarification. It appears from the available results that back-to-back VEGF inhibition is likely to be the most effective approach in treating these patients. However, it is important that the findings in PFS line up with the findings in OS in this trial. A valid critique of the Phase III trial was that data regarding follow-up treatments was available for only $10 \%$ of patients. In those $10 \%$, the investigators noted that there was a balance in both arms of subsequent therapy, in that those who received mTOR inhibition went on to receive tyrosine-kinase inhibitors in the same proportion as those receiving tyrosine-kinase inhibitors went on to receive mTOR inhibition. Unfortunately survival data is not available in the $10 \%$ of patients for whom the follow-up information was available. Questions remain about the validity and robustness of PFS as a primary end point, and when the secondary end point of OS does not line up with PFS, it is pertinent to ask whether something has not been measured subsequent to trial therapy. There is likely to be an undefined population, perhaps those with poor prognosis, for whom mTOR-inhibition is warranted in this setting. However, based on the results of our patient cohort and available Phase III data, the "off-label" use of 
temsirolimus in patients who have had previous systemic treatments should be avoided.

\section{Disclosure}

The authors report no conflicts of interest in this work.

\section{References}

1. Santoni M, Rizzo M, Burattini L, Berardi R, Carteni G, Cascinu S. Novel agents, combinations and sequences for the treatment of advanced renal cell carcinoma: When is the revolution coming? Curr Cancer Drug Targets. 2013;13(3):313-325.

2. van der Veldt $\mathrm{AA}^{1}$, Haanen JB, van den Eertwegh AJ, Boven E. Targeted therapy for renal cell cancer: current perspectives. Discov Med. 2010;10(54):394-405.

3. Garcia JA, Danielpour D. Mammalian target of rapamycin inhibition as a therapeutic strategy in the management of urologic malignancies. Mol Cancer Ther. 2008;7(6):1347-1354.

4. Atkins MB, Hidalgo M, Stadler WM, et al. Randomized phase II study of multiple dose levels of CCI-779, a novel mammalian target of rapamycin kinase inhibitor, in patients with advanced refractory renal cell carcinoma. J Clin Oncol. 2004;22(5):909-918.

5. Hudes G, Carducci M, Tomczak P, et al; Global ARCC Trial. Temsirolimus, interferon alfa, or both for advanced renal-cell carcinoma. $N$ Engl J Med. 2007;356(22):2271-2281.

6. GlaxoSmithKline. A study of pazopanib efficacy and safety in patients with advanced clear-cell renal cell carcinoma and ECOG Performance Status 2 (PaZ02). UKCRN identifier: 11827. Available from: http:// public.ukcrn.org.uk/search/StudyDetail.aspx?StudyID=11827. Accessed October 01, 2014.
7. Mackenzie MJ, Rini BI, Elson P, et al. Temsirolimus in VEGF-refractory metastatic renal cell carcinoma. Ann Oncol. 2011;22(1):145-148.

8. Weikert S, Kempkensteffen C, Busch J, et al. Sequential mTOR inhibitor treatment with temsirolimus in metastatic renal cell carcinoma following failure of VEGF receptor tyrosine kinase inhibitors. World J Urol. 2013;31(4):805-809.

9. Coppin C, Le L, Porzsolt F, Wilt T. Targeted therapy for advanced renal cell carcinoma. Cochrane Database Syst Rev. 2008;16(2):CD006017.

10. Therasse P, Arbuck SG, Eisenhauer EA, et al. New guidelines to evaluate the response to treatment in solid tumors. European Organization for Research and Treatment of Cancer, National Cancer Institute of the United States, National Cancer Institute of Canada. J Natl Cancer Inst. 2000;92(3):205-216.

11. Gerullis H, Bergmann L, Maute L, Eimer C, Otto T. Experiences and practical conclusions concerning temsirolimus use and adverse event management in advanced renal cell carcinoma within a compassionate use program in Germany. Cancer Chemother Pharmacol. 2009; 63(6):1097-1102.

12. Hutson TE, Escudier B, Esteban E, et al. Temsirolimus vs sorafenib as second line therapy in metastatic renal cell carcinoma: Phase 3 results from the INTORSECT trial. ESMO: Abstract LBA22, 2012.

13. Maroto JP, Hudes G, Dutcher JP, et al. Drug-related pneumonitis in patients with advanced renal cell carcinoma treated with temsirolimus. J Clin Oncol. 2011;29(13):1750-1756.

\section{Publish your work in this journal}

Drug Design, Development and Therapy is an international, peerreviewed open-access journal that spans the spectrum of drug design and development through to clinical applications. Clinical outcomes, patient safety, and programs for the development and effective, safe, and sustained use of medicines are a feature of the journal, which

\section{Dovepress}

has also been accepted for indexing on PubMed Central. The manuscript management system is completely online and includes a very quick and fair peer-review system, which is all easy to use. Visit http://www.dovepress.com/testimonials.php to read real quotes from published authors.

Submit your manuscript here: http://www.dovepress.com/drug-design-development-and-therapy-journal 